\title{
Adult inpatients on desmopressin: A patient safety initiative
}

\section{Bonfield $A^{1}$, Crasto $W^{2}$, Hackett $E^{3}$, Gleeson $H^{4}$}

${ }^{1}$ Department of Emergency Medicine, ${ }^{2}$ Department of Diabetes and Endocrinology, ${ }^{3}$ Pharmacy department University Hospitals of Leicester

\section{Background}

Errors made in hospitalised endocrine patients with critical medicines such as desmopressin \& hydrocortisone can undermine patient safety but are largely preventable.

\section{Methods}

We carried out a retrospective audit of adult patients admitted to the University Hospitals of Leicester NHS Trust, UK between January 2013 to June 2014, who were prescribed desmopressin for cranial diabetes insipidus (CDI) or non CDI (other medical conditions such as nocturnal incontinence) using electronic prescribing (ePMA). Safety concerns were recorded based on a scoring system according to pre-defined parameters: patient confusion, abnormalities of serum sodium levels, evidence of missed or delayed administration of desmopressin and hydrocortisone (each parameter scored 1 point).

\section{Results}

- 15 patients ( 7 males, median age 56 , range $17-85)$ totalling 31 admissions were prescribed desmopressin via ePMA

- Out of a total of 280 desmopressin prescriptions, 53 were missed $(18.9 \%)$ while 4 were delayed $(1.4 \%)$

- 8 patients had an underlying diagnosis of CDI making up 17 of the admissions

- $70 \%$ were admitted with confusion

- $35 \%$ had an abnormal sodium value

- $88 \%$ had missed/delayed desmopressin administration

- Reasons for missed/delayed: medications unavailable (64.9\%), no reason mentioned (15.8\%), patient declined $(7.0 \%)$ and nil orally or wrong timing of medication $(12.3 \%)$

- In two patients missed doses resulted in hypernatraemia

- 7 patients in non-CDI group making up 14 admissions - $64 \%$ had missed/delayed desmopressin

- Reason for missed/delayed: medications unavailable $(44.4 \%)$, patient declining $(16.7 \%)$, no reason $(11.1 \%)$ and other, e.g. nil by mouth $(27.8 \%)$

\section{Safety concerns}

Each admission was scored against 3 safety concern, those on hydrocortisone were considered to potentially have 4 safety concerns

- 5 of the $8 \mathrm{CDI}$ patients (14/17 admissions) were also on hydrocortisone

- $35.7 \%$ of these admissions had missed/delayed hydrocortisone

- CDI group had a median of 2 safety concerns and all had at least 1

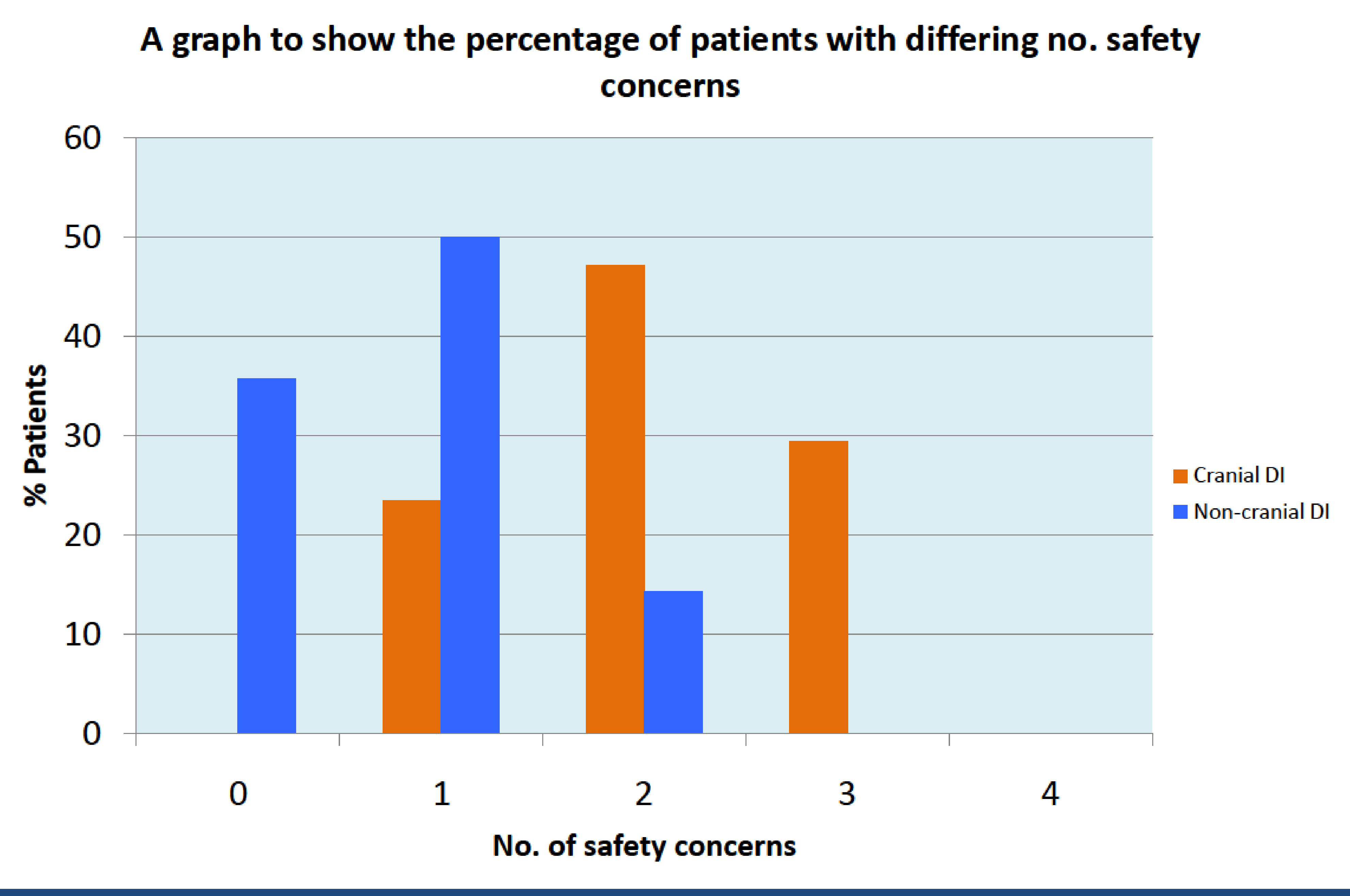

\section{Conclusion}

- Errors in prescribing \& administering desmopressin are frequent with evidence of harm

- Increased pharmacovigilance \& education surrounding the management of desmopressin in clinical areas is needed

\section{Audit recommendations}

In UHL we have undertaken 3 key strategies in order to reduce the damaging impact that such errors have on patient care:

1) To implement a system whereby the endocrine \& diabetes team are electronically notified of the majority of patients admitted on desmopressin

2) Utilising the advantages of an electronic prescribing system by warning all operators of the importance of critical medications

3) An MDT approach to the education of staff, including the development of a SUI video based around critical medication prescription \& administration

Future

A re-audit will be undertaken as soon as all of the changes proposed have been implemented. This will help to assess the effectiveness of our strategy while providing suggestions for the diabetes \& endocrine community to improve care in this important area. 